Delft University of Technology

\title{
Experiments and theory of 138 La radioactive decay
}

Quarati, FGA; Dorenbos, P; Mougeot, X.

DOI

10.1016/j.apradiso.2015.11.080

10.1016/j.apradiso.2016.01.017

Publication date

2016

Document Version

Accepted author manuscript

Published in

Applied Radiation and Isotopes

Citation (APA) Applied Radiation and Isotopes, 108, 30-34. https://doi.org/10.1016/j.apradiso.2015.11.080, https://doi.org/10.1016/j.apradiso.2016.01.017

\section{Important note}

To cite this publication, please use the final published version (if applicable).

Please check the document version above.

\section{Copyright}

Other than for strictly personal use, it is not permitted to download, forward or distribute the text or part of it, without the consent of the author(s) and/or copyright holder(s), unless the work is under an open content license such as Creative Commons.

\section{Takedown policy}

Please contact us and provide details if you believe this document breaches copyrights.

We will remove access to the work immediately and investigate your claim. 


\title{
Experiments and theory of ${ }^{138}$ La radioactive decay
}

Accepted in Applied Radiation and Isotopes, proceedings of the 20th International Conference on Radionuclide Metrology and its Applications (ICRM 2015)

\section{F.G.A. Quarati ${ }^{\mathrm{a}, \mathrm{b}, *}$, P. Dorenbos ${ }^{\mathrm{a}}, \mathrm{X}$. Mougeot $^{\mathrm{c}}$}

${ }^{a}$ AP, RST, FAME, Delft University of Technology, Mekelweg 15, 2629JB Delft, The Netherlands

${ }^{\mathrm{b}}$ Gonitec BV, J. Bildersstraat 60, 2596EJ, Den Haag, The Netherlands

c CEA, LIST, Laboratoire National Henri Becquerel, Bât. 602 PC111, CEA-Saclay 91191 Gif-sur-Yvette Cedex, France.

\begin{abstract}
We measured with unprecedented accuracy key features of the ${ }^{138}$ La radioactive decays as $\beta$ particle energy distribution from $0.5 \mathrm{keV}$ to the end-point and ratios of electron capture probabilities $P_{\mathrm{L}} / P_{\mathrm{K}}, P_{\mathrm{M}} / P_{\mathrm{K}}$ and $P_{\mathrm{M}} / P_{\mathrm{L}}$. This was achieved by making use of $\mathrm{LaBr}_{3}: \mathrm{Ce}$ and $\mathrm{CeBr}_{3}$ scintillator detectors. The advantage of the presented technique relies on the double role of $\mathrm{LaBr}_{3}: \mathrm{Ce}$ as source of ${ }^{138} \mathrm{La}$ and detector medium resulting in a relatively efficient counting statistics and unaltered $\beta$ energy detection. The experimental results are compared to advanced computational techniques and significant deviation is found below $20 \mathrm{keV}$ with the computational spectrum showing a 5\% excess of $\beta$ particle relative to the experimental spectrum at $10 \mathrm{keV}$.
\end{abstract}

Keywords: La-138; exchange effect; $\beta$ spectrum; electron capture probability ratios; $Q$ value

\footnotetext{
${ }^{*}$ Corresponding author. Tel.: + 31152785989.

E-mail address: f.g.a.quarati@tudelft.nl (F.G.A. Quarati).
} 


\section{Introduction}

Precise experiments on radioactive decays of nuclides corresponding to second forbidden unique transitions are limited by their long half-lives, $\log \mathrm{ft} \geq 12$. As a consequence, nuclear decay data available for these nuclides are potentially affected by large uncertainties. Such a limitation can be overcome in the case of ${ }^{138} \mathrm{La}$ radioactive decay which can be efficiently detected using $\mathrm{LaBr}_{3}: \mathrm{Ce}$ scintillator detectors. An overview of the characteristic of $\mathrm{LaBr}_{3}: \mathrm{Ce}$ scintillator detection systems can be found in Dorenbos et al. 2004 and Quarati et al. 2007. Thanks to the natural occurrence of ${ }^{138} \mathrm{La}\left(\sim 0.1 \%\right.$ in natural lanthanum), $\mathrm{LaBr}_{3}: \mathrm{Ce}$ scintillator detectors provide a very efficient $\beta$ energy detection, the $\beta$ being both emitted and detected within the scintillator. Moreover, based on a $0.25 \mathrm{~mm}$ range for $270 \mathrm{keV} \beta$ in $\mathrm{LaBr}_{3}: \mathrm{Ce}$, as available from NIST's ESTAR (ESTAR 1998), the $\beta$ disintegrations occurring close to the crystal surface and potentially affected by incomplete energy collection can be roughly evaluated to be a fraction of about $0.4 \%$ of the total collected $\beta$ disintegrations in the case of a $\mathrm{LaBr}_{3}$ :Ce detector size of $3^{\prime \prime} \times 3^{\prime \prime}\left(348 \mathrm{~cm}^{3}\right)$ as in our case.

Lanthanide elements are widely applied in various electronics and energy storage devices and revised nuclear decay data of ${ }^{138} \mathrm{La}$ may also lead to a more precise evaluation of ${ }^{138} \mathrm{La}$ contribution to the radioactive dose of workers and consumers and related exposure risks.

It was already demonstrated in Quarati et al. 2012 that the $\beta$ spectrum of ${ }^{138} \mathrm{La}$ substantially departs from its standard expected shape, presenting a sizable excess of $\beta$ particles at low energy. A recently developed computational technique by Mougeot et al. 2014 which calculates precisely the electron wave functions using accurate 
screened potentials and account for a correction of the atomic exchange effect is here applied to reproduce the experimentally determined shape of the ${ }^{138} \mathrm{La} \beta$ spectrum. In this article, we first present in Section 2 the newest results obtained with an improved experimental set up based on the use of a $3 " \times 3$ " $\mathrm{LaBr}_{3}: \mathrm{Ce}$ and $2^{\prime \prime} \times 2$ " $\mathrm{CeBr}_{3}$ scintillators. We then present in Section 3 the results together with the key features of the developed and applied computational method. A summary and an outlook are found in Section 4 which concludes this paper.

\section{Experimental methods and results}

Lanthanum-138 is the only naturally occurring radioactive isotope of lanthanum with $0.09 \%$ abundance and a half-life of $1.05 \times 10^{11}$ years. As a result, ${ }^{138}$ La produces in $\mathrm{LaBr}_{3}: \mathrm{Ce}$ about 1.5 counts $/ \mathrm{s} / \mathrm{cm}^{3}$, i.e. about 520 counts $/ \mathrm{s}$ in a $3^{\prime \prime} \times 3^{\prime \prime} \mathrm{LaBr}_{3}: \mathrm{Ce}$ scintillator. According to the latest Decay Data Evaluation Project (DDEP) evaluation (Bé, 2015), ${ }^{138}$ La decays via two distinct second forbidden unique transitions: (i) by electron capture $(\varepsilon)$ to the first excited state of ${ }^{138} \mathrm{Ba}$ with $65.2(6) \%$ probability; and (ii) by $\beta^{-}$decay to the first excited state of ${ }^{138} \mathrm{Ce}$ with the remaining $34.8(6) \%$ probability. A decay scheme of ${ }^{138} \mathrm{La}$ is shown in Fig. 1. In both cases, ${ }^{138} \mathrm{La}$ decay produces consequent emission of de-excitation $\gamma$-rays of $1435.816(10) \mathrm{keV}$ in ${ }^{138} \mathrm{Ba}$ ( $\varepsilon$ decay) and $788.744(8) \mathrm{keV}$ in ${ }^{138} \mathrm{Ce}\left(\beta^{-}\right.$decay). For readability, the $\gamma$-ray energies are rounded to $1436 \mathrm{keV}$ and $789 \mathrm{keV}$ in the rest of this paper. An in-coincidence acquisition of the $\beta$ spectral distribution can be performed by detecting the $789 \mathrm{keV} \gamma$ rays as schematically shown in Fig. 2 with a photograph of the present experimental setup with the two scintillators and the lead-castle used to reduce as far as possible environmental background contributions. This technique has already been detailed in Quarati et al. 2012, and the new experiment we present here differs by the larger size 
of the detectors used: a $3^{\prime \prime} \times 3^{\prime \prime} \mathrm{LaBr}_{3}: \mathrm{Ce}$ and a $2^{\prime \prime} \times 2^{\prime \prime} \mathrm{CeBr}_{3}$ scintillator, and by achieving improved counting statistics. Because of the relatively large energy range of about $300 \mathrm{keV}$ required for collecting the full $\beta$ particle spectrum, two separated acquisitions were performed, one over the $300 \mathrm{keV}$ energy range and a second with increased shaping amplifier gain over the first $50 \mathrm{keV}$ energy range. The latter acquisition allowed an unprecedented low energy cut off of $0.5 \mathrm{keV}$ and a clear detection of the electron capture events for the K, L and M shells. Fig. 3 shows two spectra both collected with the $\mathrm{CeBr}_{3}$ spectrometer. One spectrum is collected with only the $\mathrm{CeBr}_{3}$ detector inside the lead castle; the other spectrum is collected in the actual experimental configuration with the $\mathrm{LaBr}_{3}: \mathrm{Ce}$ mounted in close proximity as shown in Fig. 2. As already addressed in Quarati et al. 2012 and shown Fig. 3, the 789 $\mathrm{keV}$ full energy peak is detected on top of the Compton continuum of $1436 \mathrm{keV}{ }^{138} \mathrm{Ba}$ de-excitation $\gamma$-rays. As a consequence, electron capture peaks are always detected on top of the $\beta$ distribution because of the unavoidable presence of $1436 \mathrm{keV} \gamma$-rays Compton scattered down to an energy falling within the energy windows selected for the coincidences. However, these peaks create a well-defined interference with the $\beta$ spectrum and moreover an acquisition of the only shell peaks, i.e. without the $\beta$ particles, is possible (see Fig. 4 and Fig. 5). Therefore, the electron capture peaks interference can be taken into account by data reduction and processing. Experimental data for the two acquisitions mentioned above are plotted in Fig. 4 for the full energy range (300 keV) and in Fig. 5 for the $50 \mathrm{keV}$ energy range. In Fig. 5 is also plotted the net $\beta$ spectrum obtained by subtraction of the electron capture spectrum from the $\beta$ and electron capture spectrum. The experimental $\beta$ spectrum of Fig. 6 contains $10^{7}$ counts, of which $90 \%$ correspond to $\beta$ events and $10 \%$ to electron capture events. The acquisition lasted about three months because of the actual in-coincidence counting 
efficiency of our setup of about 1.5 events per second. It is worth noting that, although satisfactory, the counting efficiency could be dramatically improved using more or larger coincidence detectors. In fact, the total number of $\beta$ disintegration in a $3^{\prime \prime} \times 3^{\prime \prime}$ $\mathrm{LaBr} 3: \mathrm{Ce}$ are of the order of 180 per second. Gain stability of the acquisition setup was tested and exhibited a 24 hours relative variation within $\pm 0.05 \%$ while in the longer term few times larger variations were observed as shown in Fig. 7.

The presence of noise at the lower energy limit of the acquisition has been characterised using a random pulse generator. As seen in Fig. 4, the presence of noise starts to contribute only at energies below $0.4 \mathrm{keV}$, which led us to use a $0.5 \mathrm{keV}$ energy threshold. Otherwise the acquisition can be considered unaffected by noise or spurious randomly triggered events.

As most of scintillator detectors, $\mathrm{LaBr}_{3}$ :Ce presents a non-proportionality of the response (nPR) which produces observable departures from a linear energy calibration. In the case of $\mathrm{LaBr}_{3}: \mathrm{Ce}$, the nPR is well characterized by, e.g., Khodyuk and Dorenbos 2010. For instance, in the case of X-ray photons, a consequence of the $\mathrm{nPR}$ is that the $\mathrm{K}$ shell electron capture peak is not observed at its nominal energy of $37.44 \mathrm{keV}$ but rather at $35.5 \mathrm{keV}$ (Quarati et al. 2012). Similar effects are also observed for the electron $(\beta)$ response. However, in case of a continuum and relatively smooth $\beta$ distribution (as for ${ }^{138} \mathrm{La}$ ) the distortion effects due to the $\mathrm{nPR}$ are basically negligible and even when the uncertainty in the electron $\mathrm{nPR}$ is evaluated comparing two different nPR curves, one from Khodyuk and Dorenbos 2010 and the other one from Payne et al. 2011, only a relative $0.5 \%$ error is obtained. In order to present experimental spectra with the electron capture peaks at their nominal energies, all the spectra in Fig. 4, Fig. 5 and Fig. 6 are corrected for nPR. 


\section{Computational methods and results}

We present in this section the computational methods used to analyse the experimental results such as the endpoint energy of the $\beta^{-}$transition in Sec. 3.1; the improved calculation of the $\beta$ spectrum shape in Sec. 3.2; and the improved calculation of electron capture probabilities in Sec. 3.3.

\subsection{Deconvolution process for endpoint extraction}

The response of $\mathrm{LaBr}_{3}: \mathrm{Ce}$ crystal to monochromatic energies of ionizing electrons is well characterized and is dominated by Poisson statistics, which results in a Gaussian shape for which

$$
\operatorname{FWHM}(\%)=\frac{81}{\sqrt{E(\mathrm{keV})}}
$$

at the energies of interest. To obtain the measured spectrum, this response function must be convoluted with the energy spectrum of the detected $\beta$ particles. Such a Gaussian shape does not affect the global shape of the $\beta$ spectrum, except near the endpoint. Indeed, in this area of the spectrum with relatively few counts low statistic area, the limits on energy resolution may lead to data points occurring above the endpoint when they actually correspond to energies lower than the endpoint.

A deconvolution process has thus been implemented in order to extract an endpoint value from the measured spectrum free of the effect of the finite energy resolution. The algorithm described in (Magain, 1989) for noise-free data was followed using the response function of the crystal as the point spread function. Deconvolution is an iterative process where data are used at each step for evaluating how far the deconvolved distribution is from the true distribution. Thus, the deconvolution process intrinsically amplifies the statistical fluctuations of the measured spectrum at each step. Therefore, in the area of the spectrum with relatively 
few counts low statistic area considered, a compromise arises between statistical fluctuations and final uncertainty on the endpoint value.

For the deconvolution process, two different energy calibrations were used: 0.5 $\mathrm{keV} /$ channel and $0.25 \mathrm{keV} /$ channel were used for the same background subtracted experimental spectrum. On the corresponding Kurie plots, a linear fit was adjusted between $235 \mathrm{keV}$ and $265 \mathrm{keV}$. The number of iterations was increased until a stable endpoint energy and minimum uncertainty obtained. With $0.5 \mathrm{keV} / \mathrm{channel}$ energy calibration, 25 deconvolutions were needed, leading to $E_{\max }=264.1$ (33) $\mathrm{keV}$. With $0.25 \mathrm{keV} /$ channel energy calibration, 20 deconvolutions were necessary, leading to a consistent value of $E_{\max }=263.9$ (32) $\mathrm{keV}$. The mean value was adopted with the largest uncertainty and an additional uncertainty of $2.7 \mathrm{keV}$ from energy calibration was also considered. Eventually, we were able to establish an endpoint value of $E_{\max }$ $=264.0(43) \mathrm{keV}$, which has an uncertainty at least half of the latest evaluated value $E_{\max }=258(10) \mathrm{keV}$ (Wang et al., 2012).

\subsection{Beta spectrum calculation}

The ${ }^{138} \mathrm{La} \beta^{-}$decay exhibits a second forbidden unique transition. In such a case, the shape of the $\beta$ spectrum as expressed by using the Behrens's formalism is (Behrens and Bühring, 1982)

$$
\frac{d N}{d W} \propto p W q^{2} F_{0} L_{0}\left(q^{4}+\frac{10}{3} \lambda_{2} q^{2} p^{2}+\lambda_{3} p^{4}\right)
$$

where: $W=1+\frac{E}{m_{e}}$ is the total energy of the electron including its rest mass where $m_{\mathrm{e}}$ is the electron rest mass and $E$ is the kinetic energy of the $\beta$ electron; $p=\sqrt{W^{2}-1}$ is the $\beta$ particle momentum; $q=W_{0}-W$ is the corresponding momentum of the antineutrino particle; $W_{0}$ is the corresponding maximum energy of $\mathrm{W} ; F_{0} L_{0}$ is the 
Fermi function for an extended nuclear charge; and $\lambda_{\mathrm{k}}$ are energy dependent parameters calculated from $\beta$ electron wave functions. In the present publication this shape is referred to as the classical shape.

It was suggested in (Quarati et al., 2012) that the atomic screening effect should play an important role for this low maximum energy transition and should be evaluated more accurately. Thus, we extended our precise screening correction, set out for allowed transitions and leading to excellent results for the ${ }^{63} \mathrm{Ni}$ and ${ }^{241} \mathrm{Pu}$ decays (Mougeot et al., 2014), to forbidden unique transitions. The parameter $f$ within this correction was modified in order to take into account all the states that are reachable by the $\beta$ electron, arguing that the measured spectrum is a mix of all the contributions from each of these states (see more details in Mougeot et al., 2014). Throughout this work, this calculation is referred to as the improved calculation.

The calculated spectra, using $E_{\max }=264.0 \mathrm{keV}$, are compared to the measured spectrum in Fig. 6. Each theoretical spectrum has been convoluted with the response function (energy resolution and $\mathrm{nPR}$ ) of the $\mathrm{LaBr}_{3}: \mathrm{Ce}$ crystal. Classical calculation (blue dashed curve) fails to reproduce the measurement, as already highlighted in (Quarati et al., 2012). Improved calculation (light gray plain curve) clearly leads to significantly better agreement although the measured shape is not perfectly reproduced over the entire energy range. The discrepancy tends to increase below 20 $\mathrm{keV}$ down to low energies. As it has already been demonstrated in (Mougeot et al., 2014), the atomic exchange effect may be the explanation for the remaining discrepancy at low energy and also over the entire spectrum. However, it still has to be generalized to forbidden unique transitions.

In order to test the influence of the uncertainty of the maximum energy determined previously, improved calculations with $E_{\max }=259.7 \mathrm{keV}$ (green dot 
dashed curve) and $E_{\max }=268.3 \mathrm{keV}$ (green double dot dashed curve) were also performed and are shown in Fig. 6. One can observe that a shift in energy of $8.6 \mathrm{keV}$ has a significant influence on the spectrum shape; however the ${ }^{138} \mathrm{La} \beta$ spectrum is reproduced well within the data uncertainties, except below $20 \mathrm{keV}$.

\subsection{Calculation of electron capture probabilities}

The same physical process as for $\beta$ transitions underlies electron capture transitions. Identically, allowed and forbidden unique transitions can be calculated exactly. We describe here our preliminary calculations for the second forbidden unique electron capture transition from the ground state of ${ }^{138} \mathrm{La}$ to the first excited state of ${ }^{138} \mathrm{Ba}$. The transition energy was determined from the corresponding $Q$-value taken in (Wang et al., 2012): $Q^{+}=1740(34) \mathrm{keV}$.

In Behrens's formalism, the total capture probability is (Bambynek et al., 1977)

$$
\lambda \propto \sum_{\kappa_{x}} n_{\kappa_{x}} C_{\kappa_{x}} q_{\kappa_{x}}^{2} \beta_{\kappa_{x}}^{2} B_{\kappa_{x}}
$$

where: $\kappa_{x}$ is the shell quantum number that labels each atomic state; $n_{\kappa_{x}}$ is the relative occupation number; $C_{\kappa_{x}}$ is the "shape" factor as for $\beta$ transitions; $q_{\kappa_{x}}$ is the antineutrino momentum; $\beta_{\kappa_{x}}$ is the amplitude of the wave function; and $B_{\kappa_{x}}$ corresponds to the overlap and exchange corrections. To avoid the influence of the nuclear structure, ratios of relative probabilities are calculated using the following relation

$$
P_{K}+P_{L_{1}}+P_{L_{2}}+P_{L_{3}}+P_{M_{1}}+\cdots=1
$$

Overlap and exchange corrections were determined following Vatai's approach (Vatai, 1970). Contributions from all atomic shells were accounted for using the electron bound wave functions calculated as explained in detail in (Mougeot et al., 
2014). As the capture process leads to a daughter atom in an excited state, the influence of the hole on bound wave functions was taken into account by means of first order perturbation theory. Approximate evaluation of the shake-up and shake-off effects, leading to the creation of secondary vacancies, was also included following the approach described in (Crasemann et al., 1979).

In order to evaluate an uncertainty, our calculations were also performed using the lower and upper limits of the $Q$-value, even if this method could provide only a lower limit of the uncertainty. These ratios were also determined with the LOGFT program (LOGFT, 2001), widely used in nuclear data evaluations. All these theoretical predictions are compared in Table 1 with the corresponding measurements based on the data of Fig. 5. Our present improved calculations are undoubtedly in better agreement with the experimental values than the LOGFT calculations.

\section{Discussion and conclusion}

The present results confirm that the second forbidden unique $\beta^{-}$transition of ${ }^{138} \mathrm{La}$ exhibits an excess of $\beta$ particles at low energy and that the screening effect has to be taken into account to match experimental and computational energy distributions. Other key results are already available: measurement of the end-point energy; and measurements and computational evaluation of the relative probabilities of $\mathrm{K}, \mathrm{L}$ and M electron captures.

Comprehensive data reduction and analysis are on-going, more specifically to combine the two acquisition ranges into a single $\beta$ spectrum and to account for the presence of the electron capture peaks minimally affecting the uncertainties in the $\beta$ distribution. Regarding theoretical predictions, the atomic exchange effect should be 
generalized to forbidden unique transitions in order to reach the high experimental accuracy of the present measurement.

The proposed experimental technique can, in principle, be applied more widely to other nuclides. In fact, as reported by Quarati et al. 2014, a recently developed codoping technique demonstrated that scintillator performances can be enhanced by small quantities of chemical elements with lower valence than that of $\mathrm{Ce}^{3+}$, which can include radioactive isotopes other than ${ }^{138} \mathrm{La}$.

Observations of electron capture probabilities are also important for neutrino physics and more in particular on the existence of sterile neutrino, see e.g. Filianin et al. 2014. To that end, present experimental (and computational) errors appear to be too large for drawing any conclusion. However, a preliminary evaluation suggests that lowering the experimental error by 1 or 2 orders of magnitude is within reach after a moderate increase in experimental complexity, as, for instance, the use of several 3" $\times 3 " \mathrm{LaBr}_{3}: \mathrm{Ce}$ and coincidence detectors to improve counting statistics. Moreover, as discussed above, the experimental technique could be in principle applied to other nuclides, more appropriated for that measurements.

\section{Acknowledgements}

The authors wish to thank M.-M. Bé of CEA, LIST for her initiative to setup the present research collaboration and, together with P. Quarati of Politecnico di Torino and INFN Cagliari, for precious scientific advice. 


\section{References}

Bambynek, W., Behrens, H., Chen, M. H., Crasemann, B., Fitzpatrick, M. L., Ledingham, K. W. D., Genz, H., Mutterer, M., Intemann, R. L., 1977. Orbital Electron Capture by the Nucleus. Reviews of Modern Physics 49, 77-221; Erratum Reviews of Modern Physics 49, 961-962.

Bé, M.-M., et al., 2015. Table of Radionuclides -- Monographie BIPM-5, ed. CEA/LIST-LNHB, pub. BIPM, Vol. 8, to be published (available at http://www.nucleide.org/DDEP_WG/DDEPdata.htm).

Behrens, H., Bühring, W., 1982. Electron Radial Wave Functions and Nuclear Beta Decay. Clarendon, Oxford.

Crasemann, B., Chen, M. H., Briand, J. P., Chevallier, P., Chetioui, A., Tavernier, M., 1979. Atomic electron excitation probabilities during orbital electron capture by the nucleus. Physical Review C 19, 1042-1046.

Dorenbos, P., de Haas, J.T.M., van Eijk, C.W.E., 2004. Gamma ray spectroscopy with a $\varnothing 19 \times 19 \mathrm{~mm}^{3} \mathrm{LaBr}_{3}: 0.5 \% \mathrm{Ce}^{3+}$ scintillator. IEEE Trans. Nucl. Sci. NS-51, 1289-1296.

ELSTAR 1998, M.J. Berger, J.S. Coursey, M.A. Zucker and J. Chang, StoppingPower and Range Tables for Electrons, Protons, and Helium Ions, NIST Standard $\begin{array}{llll}\text { Reference } & \text { Database } & 124 & \text { (available }\end{array}$ http://physics.nist.gov/PhysRefData/Star/Text/ESTAR.html).

Filianin, P. E., Blaum, K., Eliseev, S. A., Gastaldo L., Novikov, Yu N., Shabaev, V. M., Tupitsyn, I. I., Vergados, J., 2014. On the keV sterile neutrino search in electron capture. J. Phys. G 41, 095004.

Firestone R.B., 1999. Table of Isotopes, $8^{\text {th }}$ ed., Wiley-VCH. Available from: http://ie.lbl.gov/toi.html 
Khodyuk, I.V. and P. Dorenbos, P., "Nonproportional response of $\mathrm{LaBr}_{3}: \mathrm{Ce}$ and LaC13:Ce scintillators to synchrotron x-ray irradiation", J. Phys.: Condens. Matter 22 (2010) 485402.

LOGFT Program, 2001. http://www.nndc.bnl.gov/nndcscr/ensdf_pgm/analysis/logft/. Original code from Gove, N. B., Martin, M. J., 1971. Log-f Tables for Beta Decay. Nucl. Data Tables A10, 205.

Magain, P., 1989. A Simple Deconvolution Algorithm. Proceedings of the First ESO/ST-ECF Data Analysis Workshop (April 17-19, 1989), eds. P. J. Grosberl, F. Murtagh and R. H. Warmels, ESO Conference and Workshop Proceedings No. 31, pp. 205-208.

Mougeot, X., Bisch, C., 2014. Consistent calculation of the screening and exchange effects in allowed $\beta^{-}$transitions. Physical Review A 90, 012501.

Payne, S.A., Moses, W.W., Sheets, S., Ahle, L., Cherepy, N.J., Sturm, B., Dazeley, S., Bizarri, G., Woon-Seng Choong, 2011. Nonproportionality of Scintillator Detectors: Theory and Experiment. II. IEEE Trans. Nucl. Sci. 58 3392-3402.

Quarati, F., Bos, A.J.J., Brandenburg, S., Dathy, C., Dorenbos, P., Kraft, S., Ostendorf, R.W., Ouspenski, V., Owens, A., 2007. X-ray and gamma-ray response of a 2" $\times 2 " \mathrm{LaBr} 3$ :Ce scintillation detector. Nucl. Instr. and Meth. A 574 $115-120$

Quarati, F.G.A., Khodyuk, I.V., van Eijk, C.W.E., Quarati, P., Dorenbos, P., 2012. Study of $138 \mathrm{La}$ radioactive decays using $\mathrm{LaBr}_{3}$ scintillators. Nucl. Instr. and Meth. A 683 (2012) 46

Quarati, F.G.A., Alekhin, M.S., Krämer, K.W., Dorenbos, P., 2014. Co-doping of CeBr3 scintillator detectors for energy resolution enhancement. Nucl. Instr. and Meth. A 735 655-658 
Vatai, E., 1970. On the Exchange and Overlap Corrections in Electron Capture. Nuclear Physics A 156, 541-552.

Wang, M., Audi, G., Wapstra, A. H., Kondev, F. G., MacCormick, M., Xu, X., Pfeiffer, B., 2012. The AME2012 atomic mass evaluation (II). Tables, graphs and references. Chinese Physics C 36 (12), 1603-2014. 


\section{Figure captions:}

Fig. 1. Radioactive decay scheme of ${ }^{138}$ La. Decay data are taken Bé, 2015 whereas decay scheme is adapted from Firestone, 1999.

Fig. 2. Schematic representation of the experimental technique. The $\gamma$-rays emitted by the radioactive decays of ${ }^{138} \mathrm{La}$ in the $\mathrm{LaBr}_{3}: \mathrm{Ce}$ crystal are detected by the $\mathrm{CeBr}_{3}$ crystal allowing to discriminate by coincidence technique selected events.

Fig. 3. Pulse high spectra collected with $\mathrm{CeBr} 3$ in two different configurations: 1) dashed line is $\mathrm{CeBr}_{3}$ intrinsic and natural background reduced to a minimum by the use of a lead castle; 2) plain line is the spectrum induced by $\mathrm{LaBr}_{3}: \mathrm{Ce}\left({ }^{138} \mathrm{La}\right)$ in $\mathrm{CeBr}_{3}$. The three regions of interest corresponding to selected events and used to trigger coincidence acquisition are reported. These are from left to right: 1) events of $789 \mathrm{keV}$ full energy peak detection, with a minority of Compton events from the 1436 $\mathrm{keV} \gamma$-ray; 2) only the above minority of Compton events from the $1436 \mathrm{keV} \gamma$-ray; events of $1436 \mathrm{keV}$ full energy peak detection.

Fig. 4. Acquisitions using the lower gain for energy range up to $300 \mathrm{keV}$. Spectra are only calibrated in energy and corrected for the non-proportionality response of the scintillator.

Fig. 5. Acquisitions using the higher gain for energy range up to $55 \mathrm{keV}$. Spectra are only roughly calibrated in energy and roughly corrected for the non-proportionality 
response of the scintillator. Log energy scale is to enhance the lower energy side of the plot.

Fig. 6. Comparison of the measured spectrum (dark gray dots) from the ${ }^{138}$ La beta decay with theoretical calculations. Blue dashed spectrum is from a classical calculation. Light gray bold plain spectrum was calculated with our improved screening correction and our measured $E_{\max }$. Light gray patterns are from the same calculation but with upper (268.3 keV, plain) and lower (259.7 keV, dashed) limits of $E_{\max }$ from its uncertainty.

Fig. 7. Gain stability of the setup observed in 1-week-long acquisition. The dotted lines highlight the gain variation of $\pm 0.05 \%$ typically observed for day to day acquisitions. 
Table 1: Evaluated and measured values of the relative electron capture probabilities for the K, L and M shells.

\begin{tabular}{cccc}
\hline Shell ratio & LOGFT & Experimental & Computational \\
\hline $\mathrm{L} / \mathrm{K}$ & $0.432(6)$ & $0.391(3)$ & $0.405(7)$ \\
$\mathrm{M} / \mathrm{K}$ & $0.138(5)$ & $0.102(3)$ & $0.0915(17)$ \\
$\mathrm{M} / \mathrm{L}$ & $0.320(8)$ & $0.261(9)$ & $0.226(7)$ \\
\hline
\end{tabular}




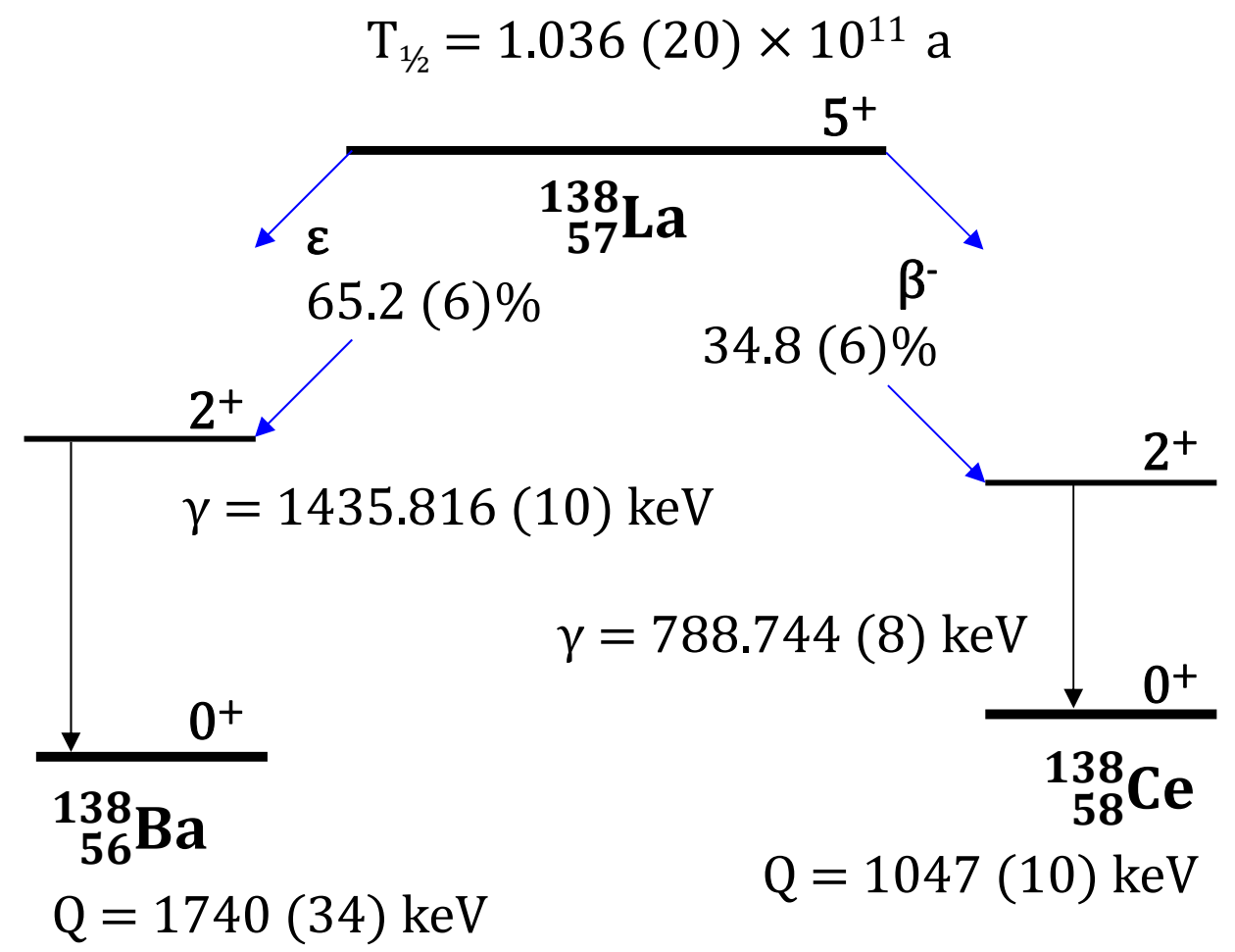

Fig. 1 
115 Quarati, Dorenbos and Mougeot: Exp. \& Th. of ${ }^{138}$ La decays Rev. June 2015
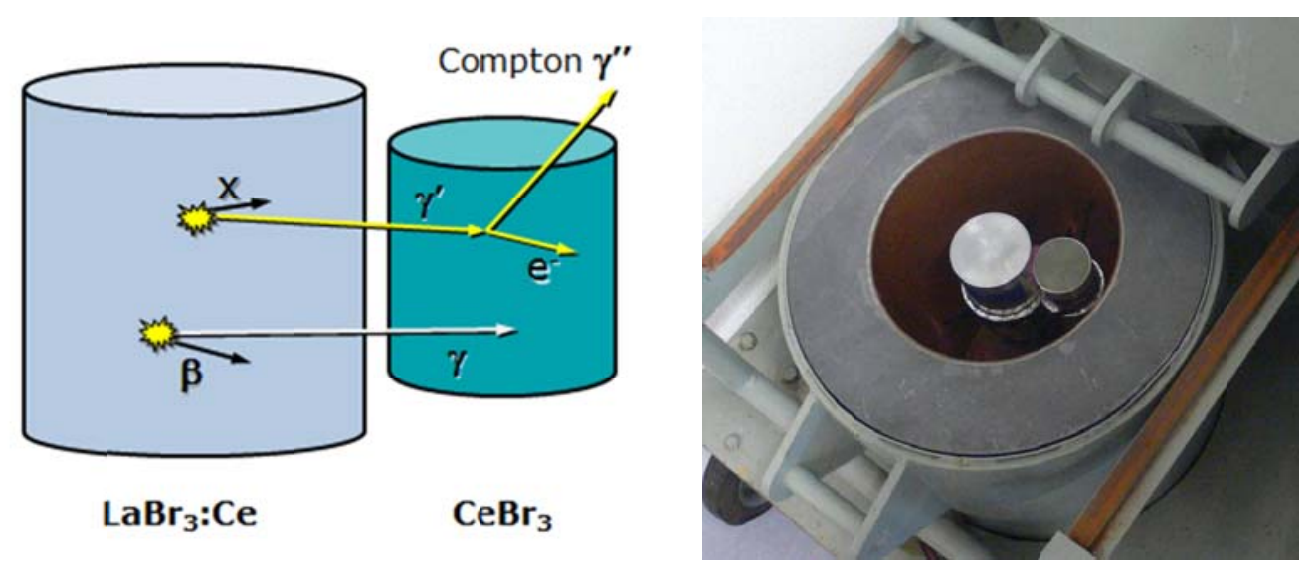

Fig. 2 
115 Quarati, Dorenbos and Mougeot: Exp. \& Th. of ${ }^{138}$ La decays Rev. June 2015

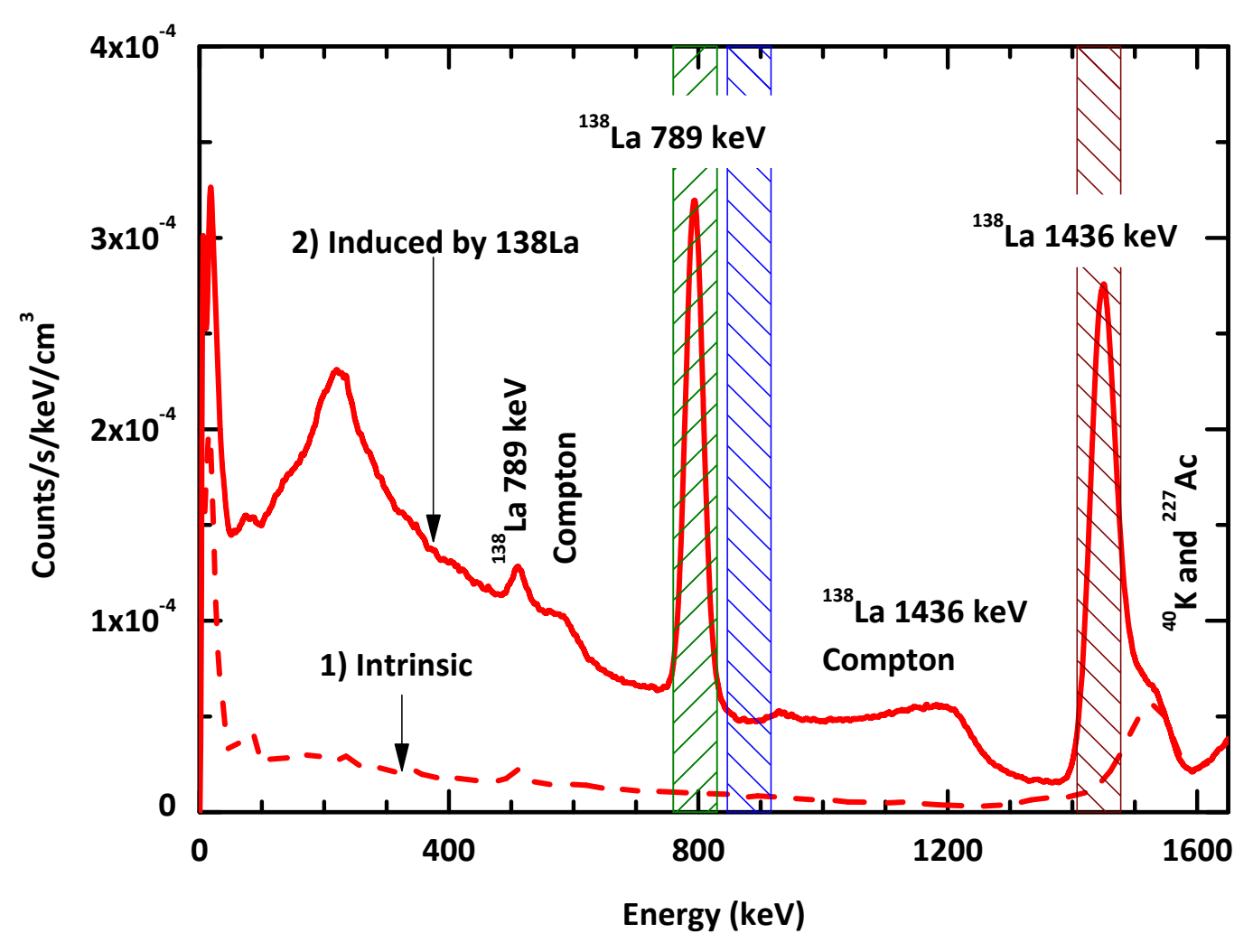

Fig. 3 
115 Quarati, Dorenbos and Mougeot: Exp. \& Th. of ${ }^{138}$ La decays Rev. June 2015

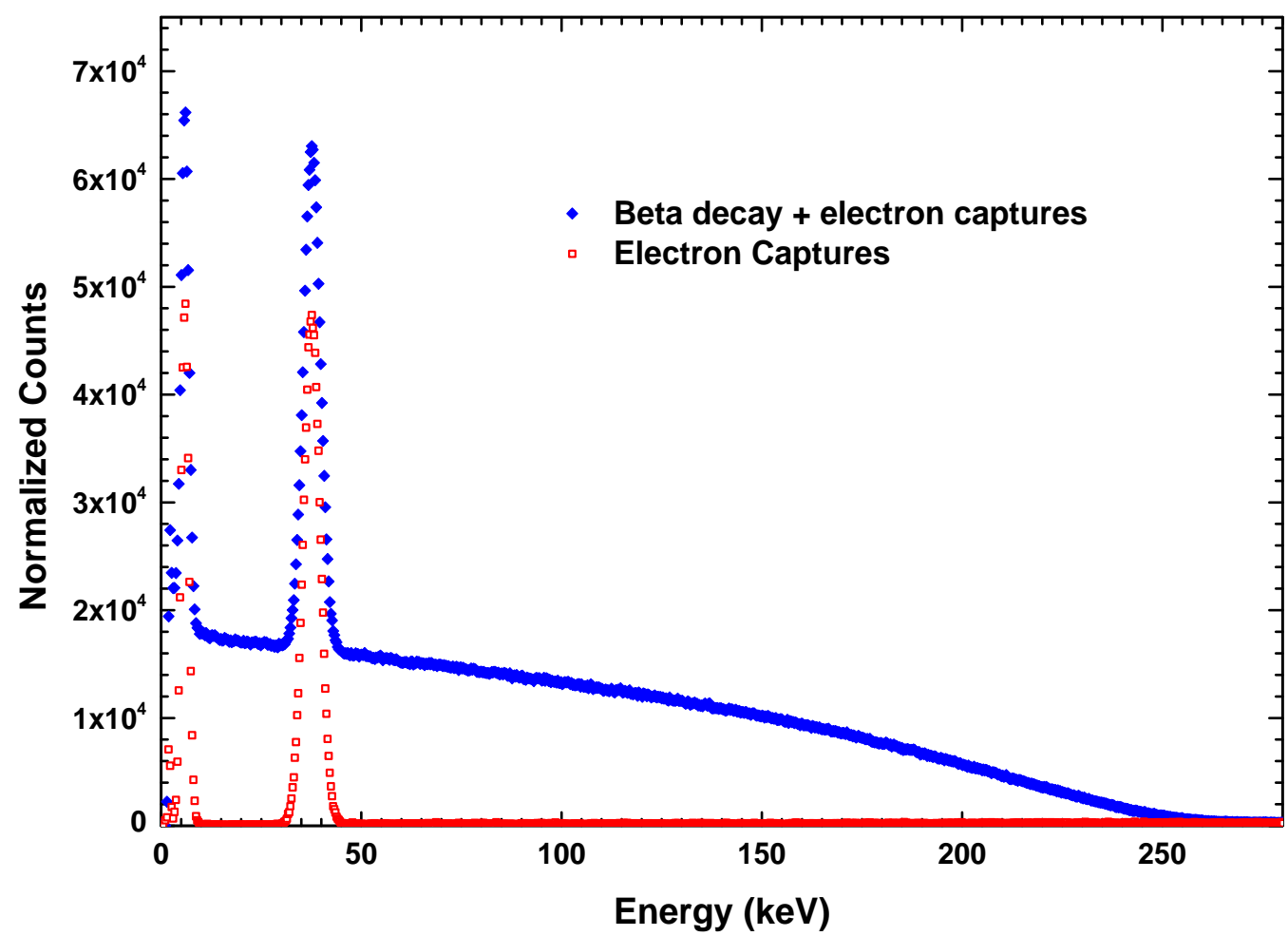

Fig. 4 
115 Quarati, Dorenbos and Mougeot: Exp. \& Th. of ${ }^{138}$ La decays Rev. June 2015

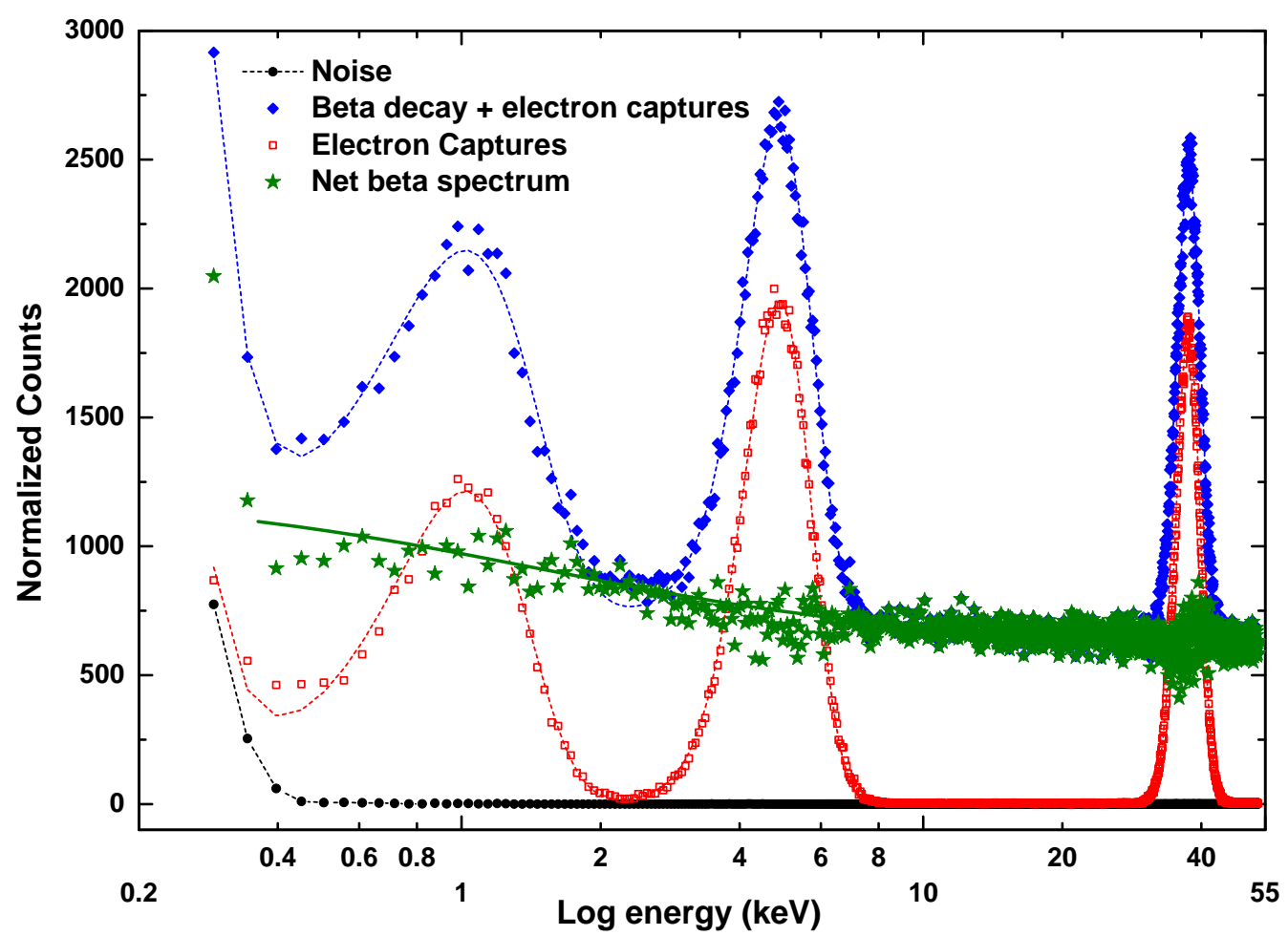

Fig. 5 
115 Quarati, Dorenbos and Mougeot: Exp. \& Th. of ${ }^{138}$ La decays Rev. June 2015

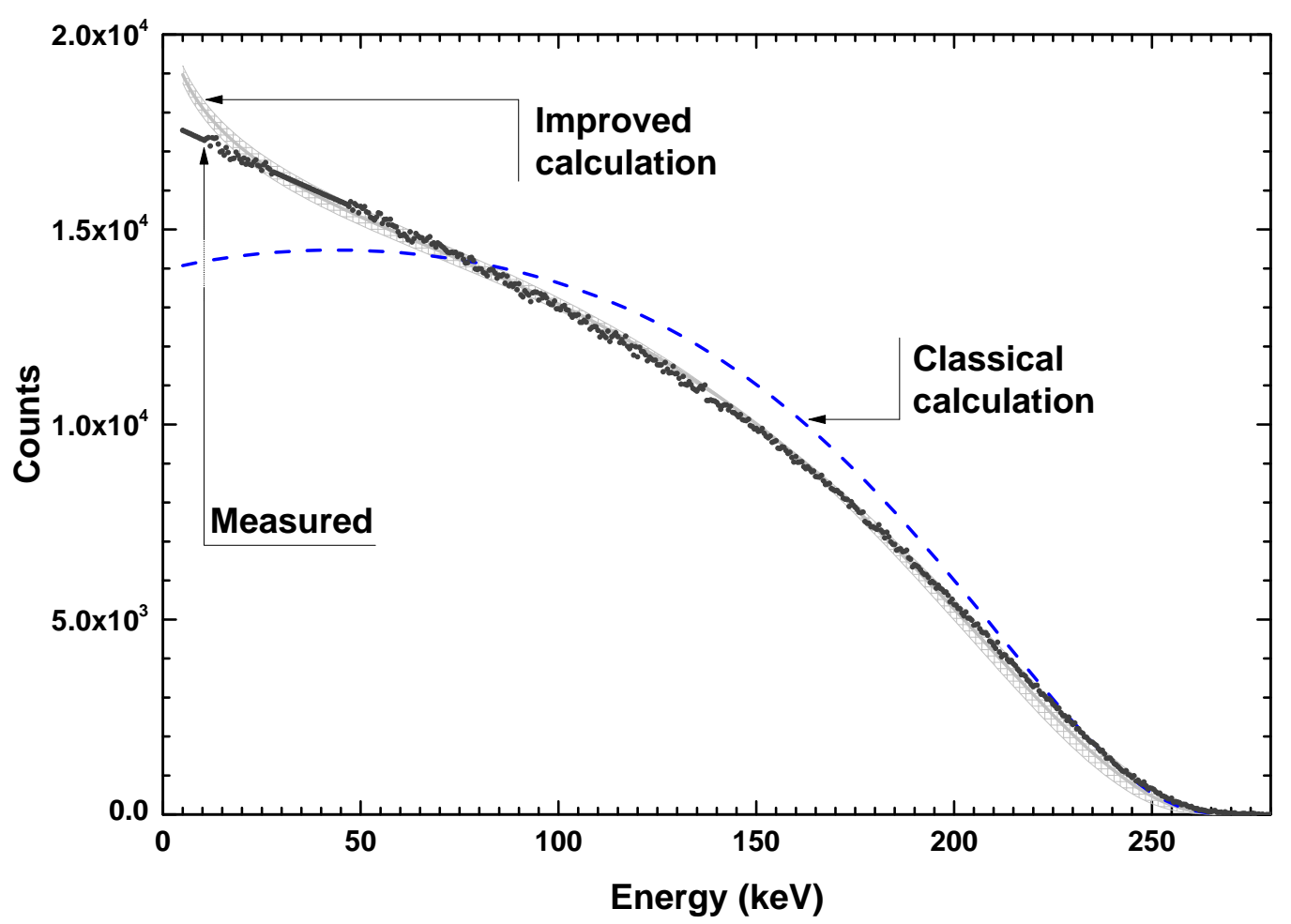

Fig. 6 
115 Quarati, Dorenbos and Mougeot: Exp. \& Th. of ${ }^{138}$ La decays Rev. June 2015

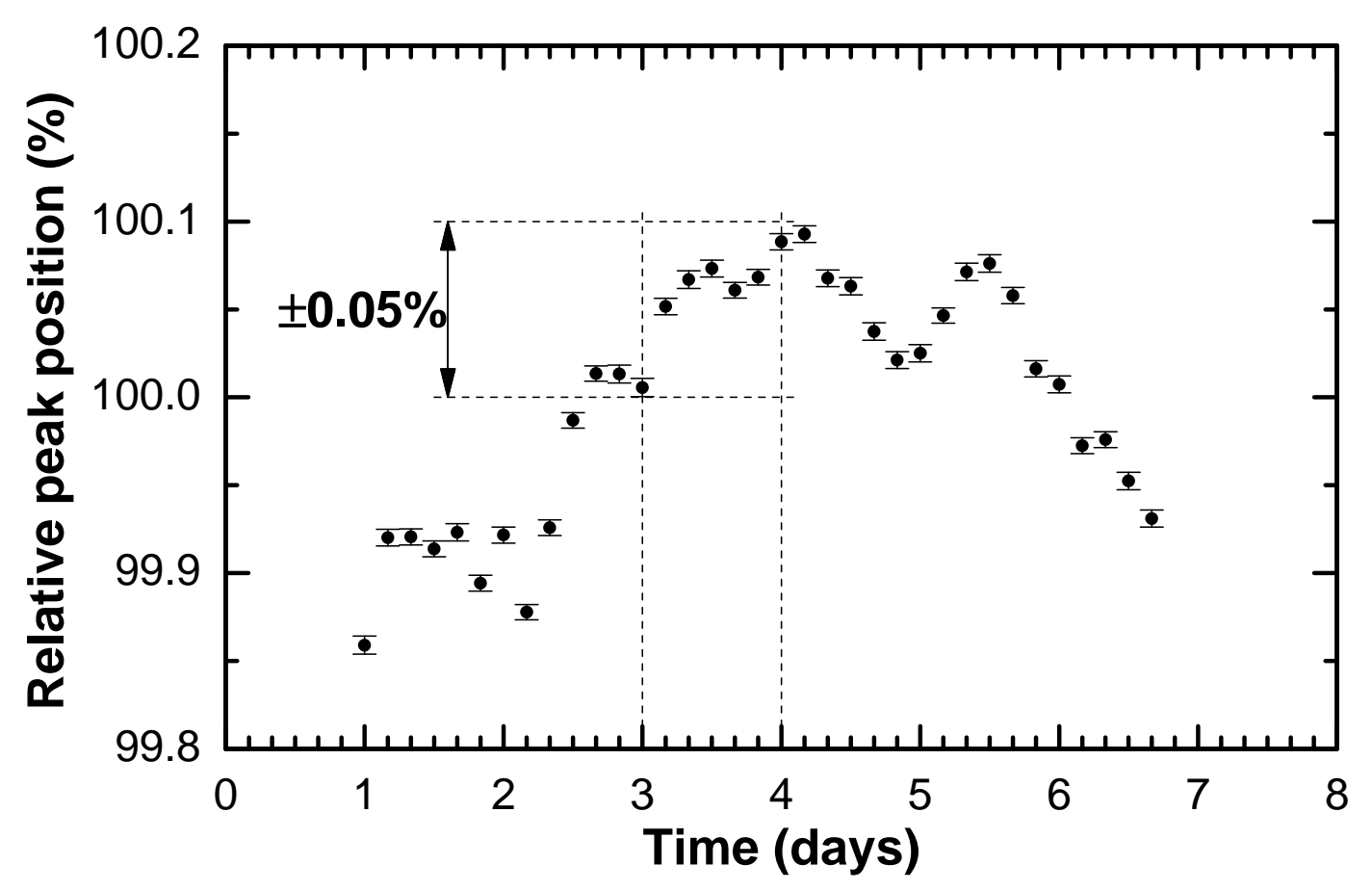

Fig. 7 